$\Rightarrow$ CHROMATIN

\section{Eukaryotic genomes in complete control}

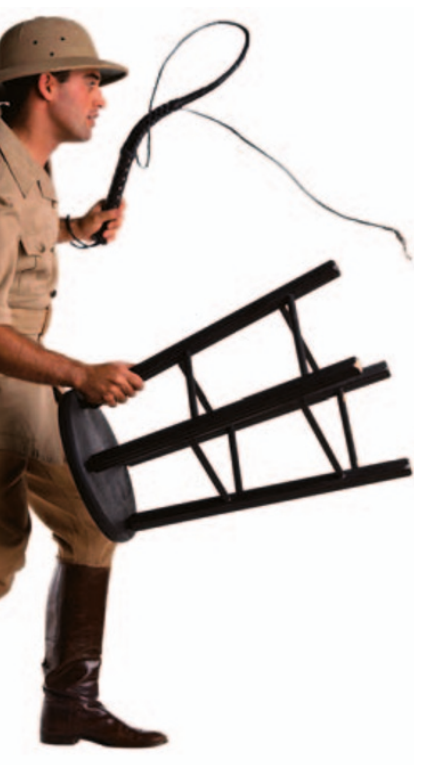

A recently published report shows that eukaryotic genomes encode preferences for nucleosome positioning, which can be modulated to facilitate specific chromosomal functions.

Nucleosomes - comprising 146 bp of DNA wrapped around a histone protein octamer - form the first level of genome compaction in eukaryotes. This organization has important implications for gene expression, because nucleosomebound DNA is less accessible to various regulatory factors than nucleosome-free DNA. But how is the positioning of the nucleosomes regulated? Could the genome itself encode a preference for their distribution?

Segal et al. addressed this question in a series of experiments that combined experimental and computational approaches. Working in yeast, the authors isolated regions of DNA that were stably wrapped around nucleosomes on a genome-wide scale. On the basis of the isolated sequences, they used a probabilistic model to predict the sequences that preferentially wrapped around the histone octamer. It is known that certain nucleotide combinations that recur with a certain frequency facilitate DNA bending; Segal et al. not only found such motifs and periodicities using their model, but, by experimentally manipulating the motif sequence and their patterns, they could enhance or reduce the binding affinity of DNA to the nucleosome in accordance with the predictions of the model.
The results also showed that the preferences of DNA for nucleosome occupancy vary from one genomic region to another. For example, centromeric sequences have the highest predicted nucleosomal occupancy; conversely, highly expressed genes such as ribosomal or transfer RNA genes show a preference for low occupancy.

Genome-wide scans showed that the high-affinity sequences occur more frequently than predicted by chance. Using a computational approach, the authors showed that various genomic regions favoured one or more nucleosome positioning patterns. Such a preference can have important functional effects; for example, a preference for a strong affinity at a transcription site would make it less accessible to transcription factors, whereas a preference for weak affinity would have the opposite effect. Using various approaches to compare in vivo nucleosome positioning data to their predictions,
The link between cancer and ageing is well established - the older animals of many species are also more likely to develop tumours. Cynthia Kenyon and colleagues now extend the link further by showing that mutations that extend lifespan in Caenorhabditis elegans also inhibit tumour growth.

To examine the links between ageing and cancer, the authors studied the interactions between mutations that affect the two processes. Mutations in the C. elegans insulin-receptor gene daf- 2 extend lifespan by more than twofold. By contrast, mutations in the gld-1 tumour-suppressor gene cause germ cells to re-enter mitosis, overproliferate and give rise to tumours that kill the animal. When the authors combined the two mutations, however, the lifespan was indistinguishable from that of the daf-2 single mutant - the tumorigenic effect of gld-1 was completely abolished.

...cell division
decreased and
apoptosis
increased in
the germ lines
of the daf-2;
gld-1 double
mutant...

How might the lack of insulin signalling prevent tumour development? The authors found that cell division decreased and apoptosis increased in the germ lines of the daf-2; gld-1 double mutant compared with the gld-1 single mutant. Interestingly, daf-2 mutations affected only germline mitosis in the tumour, and not in the normal germ lines of otherwise wildtype individuals. Also, they found that $\mathrm{p} 53$ was required for increased apoptosis and that introducing a $\mathrm{p} 53$ mutation into the double mutant reduced lifespan. This is in contrast to wild-type animals, in which p53 mutations reduce apoptosis but do not affect lifespan.

Are these effects specific to mutations in the insulin-signalling pathway, or do they occur with other lifespan-increasing mutations? Mutations in genes that affect food uptake and mitochondrial pathways also extend lifespan. Worms with such mutations, unlike daf-2 mutants, were not completely unaffected by the gld-1 mutation; however, the daf-2; gld-1 double mutants lived longer than gld-1 animals. These mutations did not affect apoptosis, but they did still reduce tumour incidence. They might therefore have more indirect downstream effects.

A connection between insulin signalling and tumour growth has also been shown in mammals, and it will be interesting to carry out equivalent experiments in mice. These results also suggest that, in contrast to $\mathrm{p} 53$, many mutations will have opposing effects on ageing and cancer.

Patrick Goymer

\section{ORIGINAL RESEARCH PAPER}

Pinkston, J. M. et al. Mutations that increase the life span of C. elegans inhibit tumor growth. Science 313, 971-975 (2006) 
Segal et al. confirmed the validity of their models and showed that 50\% of nucleosomal organization in vivo can be explained by these sequence preferences alone.

On the basis of their data, the authors suggest that by encoding weak nucleosomal affinity eukaryotic genomes might guide transcriptional machinery to functional sites. If correct, this hypothesis could also account for why some transcription factor binding sites remain unoccupied in vivo.

Although Segal et al. admit that more accurate DNA-nucleosome interaction models are needed, this report represents the first step towards integrating the effects of chromatin structure into models of gene regulation.

Magdalena Skipper

ORIGINAL RESEARCH PAPER Segal, E. et al.

A genomic code for nucleosome positioning.

Nature 442, 772-778 (2006)
$\Rightarrow$ EVOLUTION

\section{Putting flower colour on the landscape}

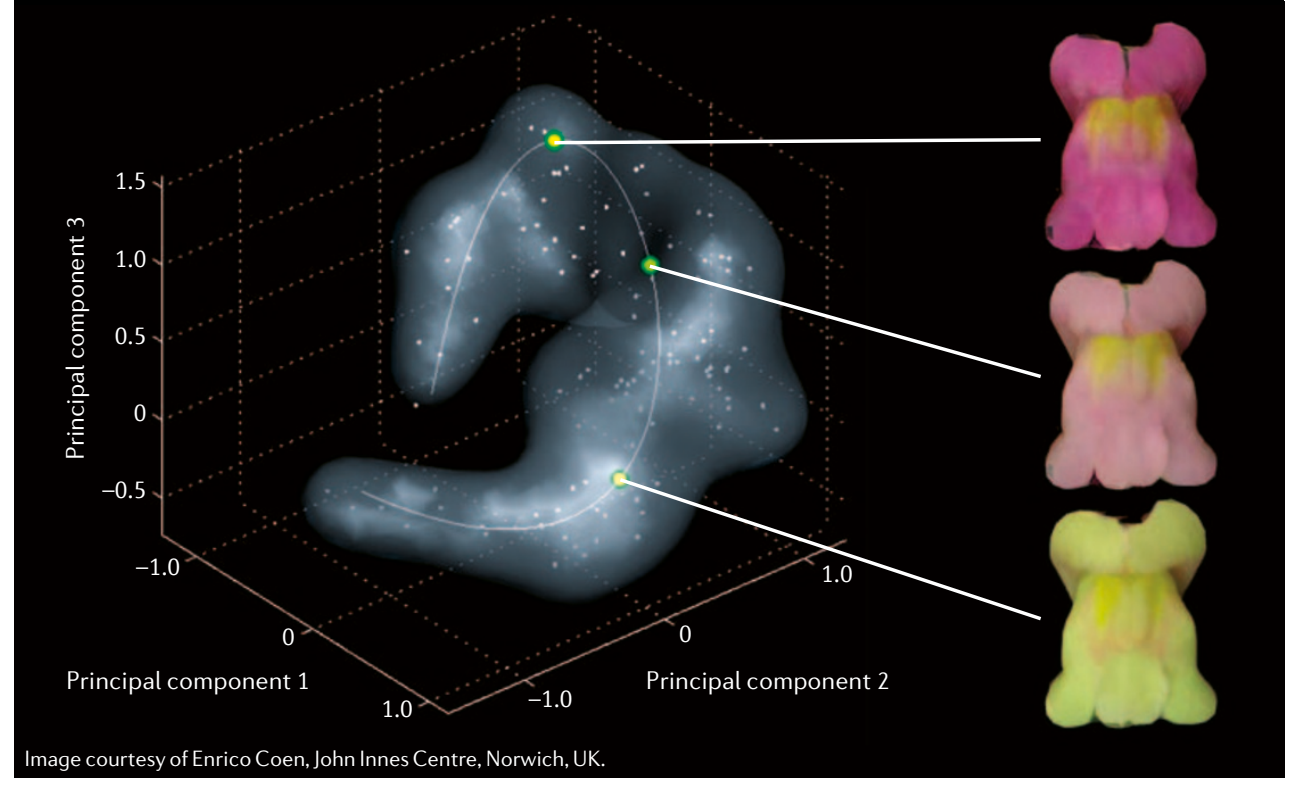

Since the 1930s, the 'adaptive landscape' has been used as a metaphor to describe the fitness of organisms in terms of an undulating surface: the fittest genotypes reside on the peaks and tower over the unfit genotypes in the valleys. But just how good is the metaphor at describing 'real life' - do new species always have to leap across a valley to reach the nearest peak? A new study of flower colour variation shows that the peaks are in fact connected by a path of transitional fitness, allowing species to 'stroll' from one peak to the other.

Accurate descriptions of adaptive landscapes have been difficult to come by. What has made it possible in this study is the use of a simple genetic system - variation in the flower colour of two species of snapdragon. The yellow-flowered Antirrhinum majus striatum and the magentaflowered Antirrhinum majus pseudomajus form a narrow hybrid zone in the region of the Pyrenees where they meet. A combination of genetic, molecular and computational studies has now been used to assess the shape of the adaptive landscape that is occupied by the genotypes in this hybrid zone.

First, the two species were assigned a genotype with respect to three genes that determine flower colour. Looking at the distribution of these alleles in the hybrid zone revealed a steep cline in the magenta and yellow morphs: if the cline was due to the neutral mixing of the alleles from the two morphs - that is, if it had arisen simply by gene flow - then alleles of genes for unrelated processes would be expected to have the same distribution. This was not the case, however, indicating that in fact selection at the colour loci was responsible for the distribution of these alleles.
It follows that the yellow and magenta morphs occupy separate adaptive peaks - in which case, how was the intervening valley crossed during the evolution of the two species? The authors addressed this question with a computational study in which they morphed the flowers of 19 Antirrhinum species to the same shape and then typed their colour patterns. When the species that were treated in this way were mapped onto genotypic space they formed a U-shaped cloud (see image): yellow and magenta occupy the extremes of the cloud, but the arm of the cloud forms a clear evolutionary path by which intermediate morphs can move from one extreme to the other, without having to dip into areas of reduced fitness. Some flower colours (such as orange) were excluded from the cloud altogether, suggesting that when the two extreme morphs meet, they might generate genotypes that 'fall out' of the high fitness region. This would account for the steep cline

This study suggests caution is necessary when interpreting the shape of adaptive landscapes: as shown here, more detailed representations might be needed to avoid mistaking isolated peaks (such as those created when you take a connected by an evolutionary path.

Tanita Casci

ORIGINAL RESEARCH PAPER Whibley, A. C. \& Langlade, N. B. et al. Evolutionary paths underlying flower color variation in Antirrhinum. Science 313, 963-966 (2006)

FURTHER READING Orr, H. A. The genetic theory of adaptation: a brief history. Nature Rev. Genet. 6, 119-127 (2005) seen in the hybrid zone. slice through the U-shaped space) for those 